\title{
Surface-induced order in liquid metals and binary alloys
}

\author{
E DiMasi $\uparrow$, H Tostmann $\ddagger$ O G Shpyrko $\ddagger$, M Deutsch§, P S Pershan $\ddagger$ and \\ B M Ocko† \\ $†$ Department of Physics, Brookhaven National Laboratory, Upton, NY 11973-5000, USA \\ \$Division of Engineering and Applied Sciences, Harvard University, Cambridge, MA 02138, \\ USA \\ § Department of Physics, Bar-Ilan University, Ramat-Gan 52900, Israel
}

Received 5 October 1999

\begin{abstract}
Measurements of the surface $\mathrm{x}$-ray scattering from several pure liquid metals $(\mathrm{Hg}, \mathrm{Ga}$ and $\mathrm{In}$ ) and from three alloys (Ga-Bi, Bi-In, and $\mathrm{K}-\mathrm{Na}$ ) with different heteroatomic chemical interactions in the bulk phase are reviewed. Surface-induced layering is found for each elemental liquid metal. The surface structure of the K-Na alloy resembles that of an elemental liquid metal. $\mathrm{Bi}-\mathrm{In}$ displays pair formation at the surface. Surface segregation and a wetting film are found for $\mathrm{Ga}-\mathrm{Bi}$.
\end{abstract}

\section{Liquid metals and surface-induced layering}

Liquid metals (LM) are comprised of charged ion cores whose Coulomb interactions are screened by a conduction electron sea. At the liquid-vapour interface, this screened Coulomb potential gives way to the weaker van der Waals interactions that prevail in the vapour. Since the potential changes so substantially across the interface, the potential gradient is high, producing a force that acts on the ions at the liquid surface as though they were packed against a hard wall. Analytic calculations and molecular dynamics simulations predict that atoms at the LM surface are stratified in layers parallel to the interface [1]. By contrast, a monotonic density profile is predicted for the vapour interface of a nonmetallic liquid.

Observation of surface layering in LM requires an experimental technique sensitive to the surface-normal density profile that can resolve length scales of 2-3 $\AA$. Specular x-ray reflectivity provides the most direct probe of the surface-normal structure. X-rays incident on the liquid surface at an angle $\alpha$ are scattered at the same angle within the reflection plane defined by the incident beam and the surface normal (figure 1(a)). The reflected intensity is directly related to the surface-normal density profile $\rho(z)$ :

$$
R\left(q_{z}\right) \propto\left|q_{z}^{-2} \int_{-\infty}^{\infty}(\partial \rho(z) / \partial z) \exp \left(\mathrm{i} q_{z} z\right) \mathrm{d} z\right|^{2} .
$$

Since $\partial \rho(z) / \partial z$ is nonzero only near the surface, $x$-ray reflectivity is sensitive to the surfacenormal structure and not to the structure of the bulk liquid. For example, surface layering with a spacing $d$ produces a quasi-Bragg peak in the reflectivity, centred at the surface-normal momentum transfer $q_{z}=(4 \pi / \lambda) \sin \alpha \approx 2 \pi / d$ [2-4].

Grazing-incidence diffraction (GID) is sensitive to the in-plane structure of the surface. The in-plane momentum transfer $q_{\|}$is probed by varying the azimuthal angle $2 \theta$ at fixed $\alpha$. 


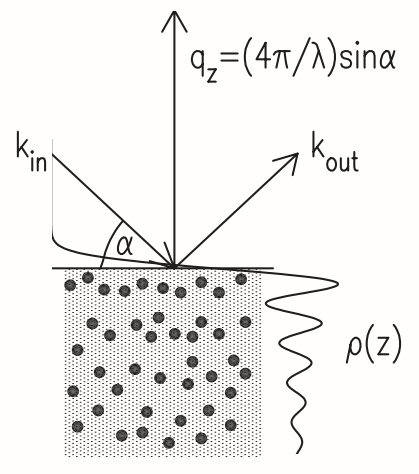

(a)

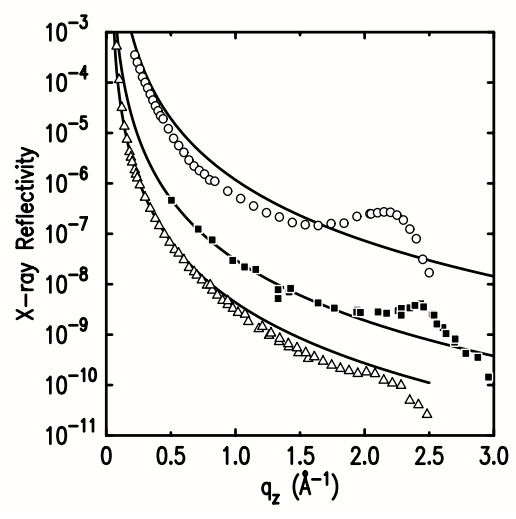

(b)

Figure 1. (a) X-ray reflectivity geometry for the liquid metal, with layering of ions producing an oscillatory density profile $\rho(z)$. (b) X-ray reflectivity for liquid $\mathrm{Hg}\left(-35^{\circ} \mathrm{C}, \circ\right), \mathrm{Ga}\left(+25^{\circ} \mathrm{C}, \mathbf{a}\right)$, and In $\left(+170{ }^{\circ} \mathrm{C}, \triangle\right)$. Solid lines: calculated Fresnel reflectivity for a flat surface. Data for $\mathrm{Ga}$ and In are shifted for clarity.

This geometry is surface sensitive when the incident angle $\alpha$ is kept below the critical angle for total external reflection, $\alpha_{c}$, thereby limiting the x-ray penetration depth [5].

For these structural studies it is essential to maintain a liquid-metal surface that is flat and clean on an atomic scale. The sample is contained either in an ultrahigh-vacuum (UHV) environment, or under a reducing atmosphere such as dry hydrogen gas, to prevent oxidation. For low-vapour-pressure, UHV-compatible metals such as $\mathrm{Ga}, \mathrm{Bi}$, and In, argon-ion sputtering is possible, and this is the most reliable way to produce an atomically clean surface [4].

Surface layering in elemental LM was first experimentally confirmed by synchrotron X-ray reflectivity measurements of liquid $\mathrm{Hg}$ [3] and $\mathrm{Ga}$ [4]. Experiments on In [6] and a number of alloys [7-10] followed. Figure 1(b) shows experimental reflectivities for three lowmelting-point elemental LM. The principle deviation from the Fresnel reflectivity calculated for a perfectly flat metal surface (solid lines) is in the broad quasi-Bragg peak centred near $q_{z}=2.2 \AA^{-1}$. These reflectivity profiles can be well described by layered density profiles decaying over several layers, shown schematically in figure 1(a).

\section{Surface structure of binary liquid alloys}

In binary alloys properties such as atomic size, surface tension, and electronic structure can be varied and should affect the details of the surface structure, thus allowing a more systematic understanding of surface layering. And since binary alloys form various ordered phases in the bulk, another interesting question arises: how does the alloy's bulk phase behaviour manifest itself at the surface, where the electronic structure, atomic coordination, and local composition are different? This question has motivated a number of studies on alloys, which have found that in general, surface layering competes with the formation of more complicated surface phases. For example, in miscible alloys the Gibbs adsorption rule predicts that the species having the lower surface energy will segregate at the surface. Observations on Ga-In [9], Ga-Sn [8], and $\mathrm{Ga}-\mathrm{Bi}$ at low $\mathrm{Bi}$ concentrations $[7,10]$ have found that surface segregation coexists with surface layering. In these alloys the first surface layer is almost entirely composed of the lower-surface-tension component (In, Sn, or Bi). By the second or third atomic layer, the bulk 
composition has been reached. In the following sections, we describe recent x-ray results for alloy surfaces which demonstrate a range of different surface-induced structural effects.

\section{1. $\mathrm{K}-\mathrm{Na}$}

Alkali metals have a simple electronic structure which can be described by ideal Fermi surfaces, and are soluble in each other with only a weak tendency towards phase formation. Since alkali metals have a very low surface tension, surface fluctuations are enhanced. These properties are expected to make the alkali metals' surface structures different from those of the main-group metals studied so far. Ideally alkali metals would be investigated under UHV conditions due to their high reactivity. However, at the melting point their high vapour pressures preclude this. By contrast, the melting point of the eutectic $\mathrm{K}_{80} \mathrm{Na}_{20}$ alloy is sufficiently low to allow UHV conditions. Due to the almost identical electron densities of the two components, when probed by $\mathrm{x}$-rays this alloy exhibits the structure of a homogeneous liquid metal. Here we present preliminary results for the eutectic $\mathrm{K}_{80} \mathrm{Na}_{20}$ alloy.

Figure 2(a) shows the $\mathrm{x}$-ray reflectivity for $\mathrm{K}_{80} \mathrm{Na}_{20}$ along with the predicted reflectivity assuming capillary-wave roughnesses (Gaussian form) of 1.2 and $1.5 \AA$. At all $q_{z}$ the reflectivity is bounded by these two curves; at lower $q_{z}$ it is better described by the $1.5 \AA$ roughness. On length scales $\gtrsim 6 \AA$ no obvious structural feature is found beyond the predicted capillary-wave roughness. The low surface tension $\left(\approx 110 \mathrm{dyn} \mathrm{cm}^{-1}\right)$ and the subsequently high roughness make it difficult to access values of $q_{z}$ large enough for directly observing a surface layering peak such as those shown for $\mathrm{Hg}, \mathrm{Ga}$, and In in figure 1(b).
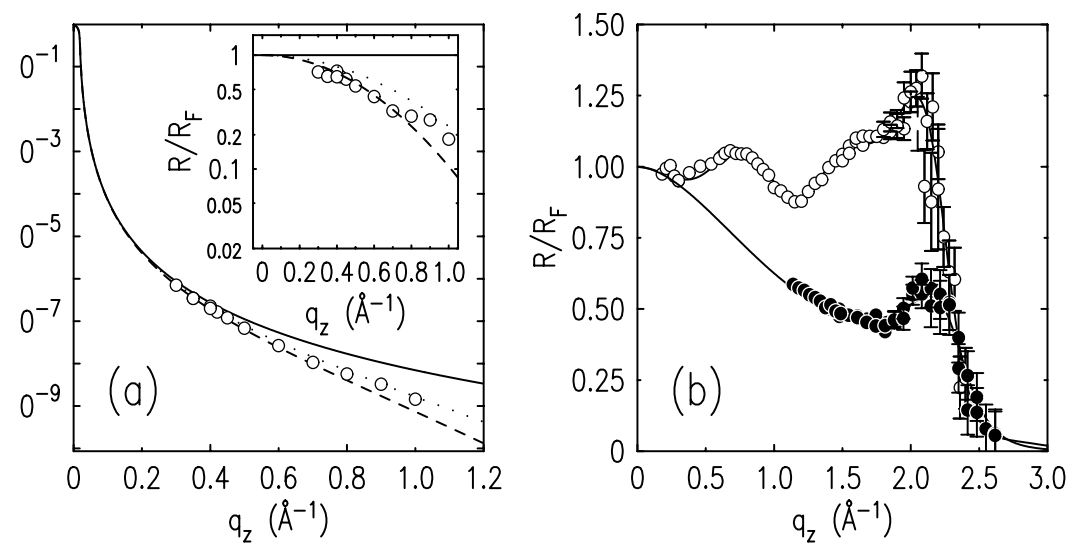

Figure 2. (a) $\mathrm{X}$-ray reflectivity for a $\mathrm{K}_{80} \mathrm{Na}_{20}$ alloy measured by integrating over a large range of $\alpha$ at fixed $\alpha+\beta$. The normalized reflectivity is shown in the inset. The dotted and dashed lines show a capillary-wave roughness with no layering with $\sigma=1.2$ and $1.5 \AA$, respectively. (b) Normalized $\mathrm{X}$-ray reflectivity of liquid $\mathrm{In}\left(+170{ }^{\circ} \mathrm{C}, \circ\right)$ and $\mathrm{Bi}_{22} \mathrm{In}_{78}\left(80^{\circ} \mathrm{C}, \bullet\right)$.

\section{2. $B i-I n$}

For systems having significant attractive interactions between unlike atoms, the surface structure is more complex. This is especially true of alloys such as Bi-In which form wellordered intermetallic phases in the bulk solid. In figure 2(b) we show the normalized reflectivity for the eutectic composition $\mathrm{Bi}_{22} \mathrm{In}_{78}$, measured at $80{ }^{\circ} \mathrm{C}(\mathrm{o})$, along with the normalized reflectivity for liquid In at $+170{ }^{\circ} \mathrm{C}(\bullet)$. The alloy exhibits a well-defined layering peak 
centred at $2.0 \AA^{-1}$ which resembles the layering peak found for pure In. In addition, the reflectivity displays a modulation with a period of about $0.9 \AA^{-1}$. This oscillation indicates that ordering at the surface occurs with a length scale nearly twice that of the inter-atomic spacing. This suggests the presence of Bi-In pairs at the surface. A full report on the phase behaviour of three different In-Bi alloys will be given elsewhere [11].

\section{3. $G a-B i$}

The Ga-Bi system is an example of an alloy with repulsive heteroatomic interactions leading to a bulk miscibility gap. Below the monotectic temperature, $T_{m o n o}=222{ }^{\circ} \mathrm{C}$, a Ga-rich liquid coexists with a solid Bi phase [12]. Due to its lower surface energy, a Bi monolayer is expected to segregate at the surface of the Ga-rich liquid. The Bi concentration in the Ga-rich phase increases with increasing temperature as long as the Ga-rich liquid coexists with the solid Bi phase. Above $T_{m o n o}$, the Ga- and Bi-rich liquids comprise a binary mixture with critical demixing, and the alloy is consequently predicted to undergo a wetting transition at a characteristic temperature $T_{w}$ below the critical temperature $T_{c}$ [13]. In the case of $\mathrm{Ga}-\mathrm{Bi}$, a macroscopically thick Bi-rich wetting film develops for $T>T_{w}$, floating on the Ga-rich liquid despite its higher density [12].

Normalized x-ray reflectivity spectra, $R / R_{F}$, for $\mathrm{Ga}-\mathrm{Bi}$ at $35{ }^{\circ} \mathrm{C}$ and $228{ }^{\circ} \mathrm{C}$ are shown in figure 3(a) versus $q_{z}$, along with the profile for pure $\mathrm{Ga}$ at room temperature. At $35^{\circ} \mathrm{C}$ the normalized reflectivity has a broad maximum at $q_{z} \approx 1 \AA^{-1}$. As suggested by Lei et al [7], this is consistent with a density profile with a thin, high-density monolayer of Bi.
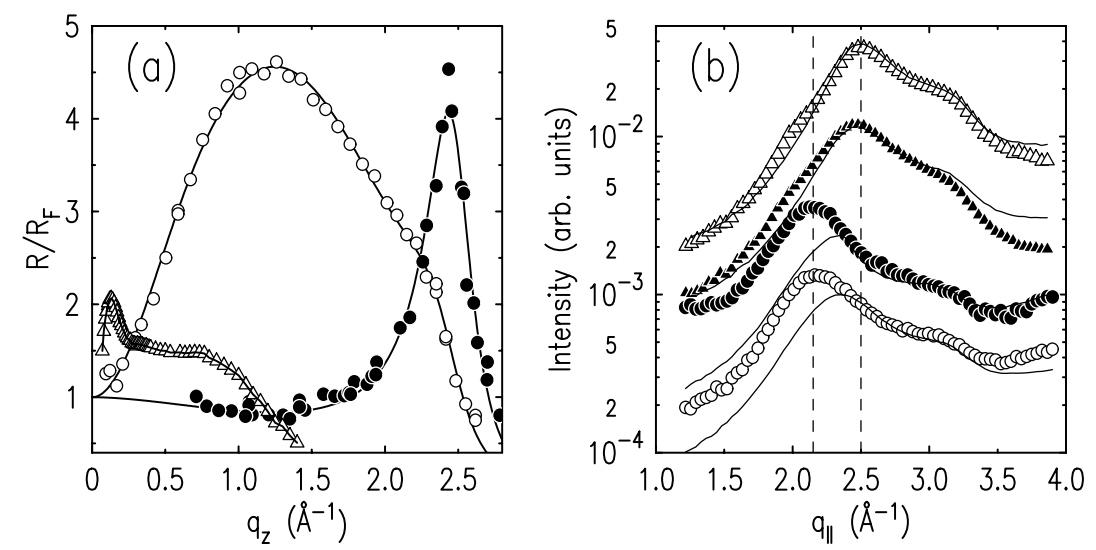

Figure 3. (a) Normalized $\mathrm{x}$-ray reflectivity of liquid $\mathrm{Ga}\left(+25^{\circ} \mathrm{C}, \bullet\right)$, and on the $\mathrm{Ga}-\mathrm{Bi}$ two-phase coexistence curve at $35{ }^{\circ} \mathrm{C}(\circ)$ and $228{ }^{\circ} \mathrm{C}(\triangle)$. (b) Grazing-incidence diffraction from Ga-Bi at $150{ }^{\circ} \mathrm{C}(\triangle), 205{ }^{\circ} \mathrm{C}(\Delta), 228{ }^{\circ} \mathrm{C}(\bullet)$, and $255^{\circ} \mathrm{C}(\circ)$ at $\alpha=0.08^{\circ}$. The solid lines show corresponding profiles for $\alpha=0.30^{\circ}$ where the bulk is predominantly sampled, and have been normalized at $2.5 \AA^{-1}$ to the GID curves. The data were acquired using Soller slits, of $0.05 \AA^{-1}$ FWHM.

We have fitted the reflectivity profiles to simple density profiles using equation (1). The fitted reflectivities are shown in figure 3 (a) (solid lines). At $+35^{\circ} \mathrm{C}$ the local density profile exhibits a top-layer density which is about 1.5 times higher than the Ga bulk liquid density. The $3.4 \pm 0.2 \AA$ layer spacing between the surface and the adjacent Ga layer obtained from the fits is much larger than the $2.5 \pm 0.1 \AA$ Alayer spacing obtained in liquid gallium. The data show that the surface layer has a higher density than in the underlying Ga-rich subphase, confirming 
the surface segregation of a Bi monolayer.

The reflectivity at $228^{\circ} \mathrm{C}$ is markedly different: a sharp peak in $R\left(q_{z}\right)$ has emerged, centred around $0.13 \AA^{-1}$. The peak at small $q_{z}$ indicates the presence of a thick surface layer with a density greater than that of the bulk subphase. The absence of additional oscillations following the sharp peak suggests that the boundary between the two regions must either be diffuse or rough. The persistence of the broad maximum at $q_{z} \approx 0.75 \AA^{-1}$ indicates that Bi monolayer segregation coexists with the newly formed wetting film. Fits to a simple two-box model yield a film thickness of $30 \AA$ consistent with ellipsometry results [12], and a surface density consistent with the high-density liquid phase of the bulk alloy. The temperature-dependent reflectivity will be reported elsewhere [14].

In figure 3(b) GID data are shown from the same $\mathrm{Ga}-\mathrm{Bi}$ alloy in the temperature range from 150 to $255^{\circ} \mathrm{C}$. In the liquid Ga-rich phase the $\mathrm{Bi}$ concentration ranges from 3.3 at. $\%$ to 17.8 at.\%. At each temperature data were taken above and below the critical angle $\alpha_{c}=0.14^{\circ}$. At $\alpha=0.08^{\circ}$ (symbols), the $\mathrm{x}$-ray penetration depth is limited to about $30 \AA$ or about ten atomic layers, maximizing the contribution from the surface. Measurements at $\alpha=0.30^{\circ}$ (solid lines) predominantly sample the bulk.

At $150{ }^{\circ} \mathrm{C}$ the bulk liquid scattering is dominated by the structure of pure $\mathrm{Ga}$ since the Bi concentration is low. The broad peak at $q_{\|}=2.5 \AA^{-1}$ and the shoulder on the high-angle side of the peak are in agreement with the bulk liquid-Ga structure factor [15]. There is no evidence for a peak or shoulder at the position corresponding to the first peak of the Bi liquid structure factor at $q_{\|} \approx 2.2 \AA^{-1}$. This is expected since the surface regime is so much smaller than the bulk volume sampled. For $\alpha=0.08^{\circ}<\alpha_{c}$, a shoulder appears on the low- $q$ side of the gallium liquid peak, due to enhanced sensitivity to the Bi surface monolayer. Between $150^{\circ} \mathrm{C}$ and $205^{\circ} \mathrm{C}$ there is little change in the GID data, except a slight increase in the shoulder associated with the Bi monolayer.

Above $T_{m o n o}$ there is a dramatic change in the GID profiles. In figure 3(b), GID data are shown at $228{ }^{\circ} \mathrm{C}$ and $255^{\circ} \mathrm{C}$. In both cases, for $\alpha>\alpha_{c}$ the peak has shifted to $q_{\|} \approx 2.3 \AA^{-1}$ from the $2.5 \AA^{-1}$ peak position found at lower temperatures. This results from the much higher Bi concentration in the bulk at the higher temperatures and the larger atomic size of Bi. Even more dramatic is the shift in the peak position for $\alpha<\alpha_{c}$ where the peak is at $2.15 \AA^{-1}$. Thus, the surface region contains considerably more Bi than the underlying bulk alloy. This finding is consistent with the wetting layer observed in the x-ray reflectivity measurements.

\section{Acknowledgments}

This work was supported by the US DOE Grant No DE-FG02-88-ER45379 and the US-Israel Binational Science Foundation, Jerusalem. Brookhaven National Laboratory is supported by US DOE Contract No DE-AC02-98CH10886. HT acknowledges support from the Deutsche Forschungsgemeinschaft.

\section{References}

[1] Rice S A 1996 J. Non-Cryst. Solids 205-207 755 and references therein

[2] Bosio L, Cortes R, Defrain A and Oumezine M 1984 J. Non-Cryst. Solids 61+62 697

[3] Magnussen O M, Ocko B M, Regan M J, Penanen K, Pershan P S and Deutsch M 1995 Phys. Rev. Lett. 744444 DiMasi E, Tostmann H, Ocko B M, Pershan P S and Deutsch M 1998 Phys. Rev. B 58 R13 419

[4] Regan M J, Kawamoto E H, Lee S, Pershan P S, Maskil N, Deutsch M, Magnussen O M, Ocko B M and Berman L E 1995 Phys. Rev. Lett. 752498

Regan M J, Pershan P S, Magnussen O M, Ocko B M, Deutsch M and Berman L E 1996 Phys. Rev. B 549730

[5] Eisenberger P and Marra W C 1981 Phys. Rev. Lett. 461081 
[6] Tostmann H, DiMasi E, Shpyrko O G, Pershan P S, Deutsch M and Ocko B M 1999 Phys. Rev. B 59783

[7] Lei N, Huang Z and Rice S A 1996 J. Chem. Phys. 1044802

[8] Lei N, Huang Z and Rice S A 1997 J. Chem. Phys. 1074051

[9] Regan M J, Pershan P S, Magnussen O M, Ocko B M, Deutsch M and Berman L E 1997 Phys. Rev. B 5515874

[10] Tostmann H, DiMasi E, Shpyrko O G, Pershan P S, Ocko B M and Deutsch M 1998 Ber. Bunsenges. Phys. Chem. 1021136

[11] DiMasi E et al 1999 in preparation

[12] Nattland D, Müller S C, Poh P D and Freyland W 1996 J. Non-Cryst. Solids 205-207 772

[13] Cahn J W 1977 J. Chem. Phys. 663667

[14] Tostmann H, DiMasi E, Shpyrko O G, Pershan P S, Ocko B M and Deutsch M D 1999 Phys. Rev. Lett. submitted

[15] Narten A H 1972 J. Chem. Phys. 561185 